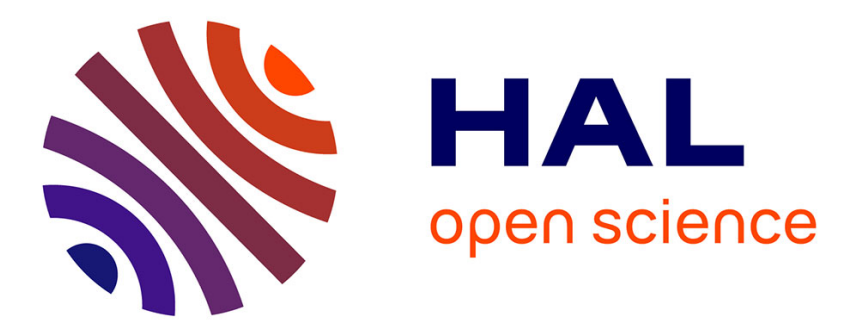

\title{
Tectonique de couverture et discordance tectonisée en Armorique
}

Claude Klein

\section{To cite this version:}

Claude Klein. Tectonique de couverture et discordance tectonisée en Armorique. Bulletin de l'Association de géographes français, 1957, 263-264, pp.29-39. 10.3406/bagf.1957.7527. insu02284872

\section{HAL Id: insu-02284872 \\ https://hal-insu.archives-ouvertes.fr/insu-02284872}

Submitted on 4 Mar 2021

HAL is a multi-disciplinary open access archive for the deposit and dissemination of scientific research documents, whether they are published or not. The documents may come from teaching and research institutions in France or abroad, or from public or private research centers.
L'archive ouverte pluridisciplinaire HAL, est destinée au dépôt et à la diffusion de documents scientifiques de niveau recherche, publiés ou non, émanant des établissements d'enseignement et de recherche français ou étrangers, des laboratoires publics ou privés.

\section{()ㅜ) $\Theta$}

Distributed under a Creative Commons Attribution - NoDerivatives| 4.0 International 


\section{Tectonique de couverture et discordance tectonisée en Armorique} Cl. Klein

\section{Citer ce document / Cite this document :}

Klein Cl. Tectonique de couverture et discordance tectonisée en Armorique. In: Bulletin de l'Association de géographes français, N²63-264, 34e année, Janvier-février 1957. pp. 29-39;

doi : https://doi.org/10.3406/bagf.1957.7527

https://www.persee.fr/doc/bagf_0004-5322_1957_num_34_263_7527

Fichier pdf généré le 25/04/2018 
Communication écrite de M. C. KLEIN

\section{Tectonique de couverture et discordance tectonisée en Armorique}

Le rôle du socle dans la tectonique de sa couverture sédimentaire est aujourd'hui au centre des préoccupations de nombreux chercheurs. En étudiant les marges armoricaines, nous avons été amené à constater cependant que les schémas classiquement admis ne paraissaient pas rendre compte de manière satisfaisante de la tectonique de la région. Ce désaccord peut d'ailleurs s'expliquer; mais pour mieux faire apparaître les caractères originaux du socle armoricain et de sa couverture paléozoïque au moment de l'orogenèse hercynienne, nous rappellerons d'abord brièvement les termes du problème lorsqu'il se pose sous son aspect classique. Nous chercherons alors à définir, en nous appuyant sur des faits concrets, comment le socle briovérien paraît avoir réagi sur sa couverture schisto-gréseuse discordante et comment une tectonique de couverture d'un genre très spécial est issue de l'épreuve mécanique des mouvements hercyniens.

\section{I. - Socle Rigide et couverture souple SÉParés par une assise plastique}

C'est à ces trois termes qu'on peut réduire, sans les trahir, les données du problème tectonique face auquel on se trouve ordinairement placé dans les régions hercyniennes masquées par une'couverture mésozoïque.

$1^{\circ}$ Le socle n'est certes pas homogène. Il comporte des terrains sédimentaires conservés dans les racines de synclinaux pincés (carbonifère le plus souvent) ou dans des poches d'effondrement profondes et localisées (permien).114ais les roches cristallines (granites, migmatites, ectinites) l'eumportent très largement. Le socle, induré par la montée des batholites (granitisation) ou par la mise en place des complexes cristallophylliens (migmatisation), beaucoup plus que par l'effet des contraintes mécaniques enregistrées, se caractérise par sa grande rigidité. A de nouvelles sollicitations mécaniques, il ne peut généralement répondre qu'en se gauchissant ou en se cassant.

$2^{\circ}$ La couverture discordante s'est déposée au sein de mers épicontinentales succédant aux épisodes continentaux, puis lagunaires de l'époque post-hercynienne (permo-trias). Des assises alternativement argilo-marneuses et calcaires se sont superposées et constituent un ensemble relativement souple, susceptible de se plisser.

$3^{\circ}$ Et ceci d'autant plus aisément qu'un coussinet plastique, ordinairement constitué par les assises gypso-salines du Trias 
moyen et supérieur, se rencontre à la base de la série mésozoïque. C'est au niveau de l'horizon plastique qu'a lieu le décollement principal et non nécessairement au niveau de la discordance basale. Ainsi G. Castany note qu'en Tunisie «le grès bigarré est solidaire du substratum primaire»(1). D'autres disharmonies apparaissent au sein de la couverture : au niveau de l'Oxfordien dans le Jura, au niveau du Lias schisteux, des marnes callooxfordiennes ou des marnes néocomiennes dans les Préalpes du Nord (2). Mais un intérêt particulier s'attache naturellement à la disharmonie qui apparaît au nivcau ou au voisinage de la surface de discordance post-hercynienne.

Grâce à cette possibilité de décollement, la couverture est donc susceptible, pourvu que les mouvements du sol le lui permettent, de se déformer d'une manière relativement autonome. Une tectonique de couverture, superposée à la tectonique propre du socle, peut apparaître. Cependant, les recherches récentes ont montré qu'il existe des degrés dans cette indépendance tectonique de la couverture et qu'il y a lieu de tenir compte du comportement du socle dont la trame tectonique peut s'exprimer, de manière parfois décisive, dans la couverture elle-même. On peut, en simplifiant, distinguer trois cas :

a) le jeu des voussoirs du socle détermine un ancrage de la couverture sédimentaire discordante et la tectonique de la couverture n'est qu'une expression passive et fidèle de la tectonique du socle. Ainsi, P. Fallot, qui ne pense pas que l'hypothèse de la gravité, appliquée par Lugeon au Jura, soit transposable dans le Bassin Parisien, écrit : "Les horsts et les grabens qui affectent simultanément bâti et série secondaire ont réalisé entre eux une sorte d'assemblage à queue d'aronde; et un glissement du complexe récent ainsi ancré sur le socle me paraît fort difficile sinon impossible à concevoir... »;

b) le jeu des compartiments du socle limite le glissement de la couverture et oriente ses déformations, mais un style tectonique propre apparaît à l'intérieur de chaque compartiment. L'indépendance de la couverture n'est encore que partielle, mais elle est déjà effective. "La surface de chacun des voussoirs en mosaïque formant le Jura s'incline différemment sous l'action d'une déformation du socle et la couverture glisse sur les surfaces inclinées du socle : c'est un glissement gravitationnel localisé...»C'est le style comtois de L. Glangeaud ; on l'observe dans le Jura externe avec son cortège de failles-plis, de pincées, de lanières (3) ;

c) si l'on passe des régions à couverture mince ( $a$ et $b$ ) aux

(1) G. Castany : Les grands traits structuraux de la Tunisic. Bull. Soc. Géol. de France, 1954, p. 151 .

(2) J. Masseport : Le Sillon Alpin. Rev. Géog. Alp., 1955, p. 810.

(3) L. Glangeaud : Les caractères structuraux du Jura. B.S.G.F., 1949. 
régions à couverture épaisse, l'influence du socle, immédiate dans les deux premiers cas, devient moins évidente. La couverture décollée et glissée s'est déformée suivant un style original très largement - mais non totalement - affranchi des déformations du socle profond.

Ainsi le style haut-jurassien du faisceau helvétique (L. Glangeaud) : le socle n'a qu'une influence médiate.

La mise en place de ces dispositifs structuraux est le résultat de deux catégories de mouvements :

- d'une part, un jeu vertical des compartiments du socle rigide qui, devenu inapte aux déformations souples, s'est cassé (à l'oligocène en ce qui concerne le Jura)';

- d'autre part, un écoulement disharmonique de la couverture décollée, à l'intérieur de chaque voussoir, au niveau des assises plastiques de la base (à l'époque mio-pliocène pour le Jura).

Cette interférence est clairement exprimée par L. Glangeaud (4) : «Le Jura est issu de l'interférence d'une tectonique de fond, avec des jeux indépendants de la couverture soumise à des poussées tangentielles... »

Il faut d'ailleurs observer à propos de ces poussées tangentielles que $\mathrm{L}$. Glangeaud les interprète bien davantage comme l'effet «de poussées profondes du socle, de jeux de socle ayant exhaussé le faisceau helvétique» que comme le résultat de l'avancée des nappes préalpines sur la molasse suisse, comme le pensait Lugeon. L'accent est donc nettement mis sur le rôle prépondérant des mouvements verticaux.

Les observations de G. Castany en Tunisie vont très exactement dans le même sens. "L'Átlas présente en Tunisie une tectonique de couverture conditionnée par la structure du socle profond... le décollement principal a lieu au niveau du Trias moyen... Le complexe salifère triasique joue le rôle de lubrifiant... $\gg(5)$.

Ce rôle du socle n'est pas moins décisif en Armorique, mais il paraît s'être exprimé de manière bien différente, car les données du problème ne se posent pas dans les mêmes termes

II. - LE « SOCLE 》 BRiovérien et SA Couverture paléozoígue

Il ne s'agit pas, au demeurant, d'examiner le comportement du socle armoricain vis-à-vis de la couverture mésozoïque, mars celui du «socle» briovérien et de sa couverture paléozoĩque discordante.

(4) L. Glangeaud et Schneggans : Caractères généraux du style jurassien... Sté Géol. de Belg., t. 73, 1949-1950. C.R. des Sessions extraordinaires.

(5) G. Castany : Les grands traits structuraux de la Tunisie. B.S.G.F., 1954, p. 151. - Tectonique et orogenèse de la Tunisie, in Cong. Géol. Internat., Alger, 1952. 
$1^{\circ}$ Le socle briovérien. Les cartes géologiques montrent que les terrains cristallins en affleurement ne couvrent guère plus du quart de la superficie. Certes, leur présence en profondeuı n'est pas contestable ; mais il a été prouvé - et les cartes en portent témoignage - que la plupart de ces roches se sont mises en place au cours de l'orogenèse hercynienne: les batholites bretons ont, en effet, digéré les séries sédimentaires siluriennes, dévoniennes et dinantiennes au milieu desquelles ils apparaissent (style Pacifique).

Il est vrai que les travaux récents - mais là encore le témoignage des cartes aurait pu être invoqué — ont remis à l'honneur les vues d'Oehlert sur l'âge antécambrien de très nombreux granites normands. C'est imprudemment que les conclusions de Ch. Barrois sur l'âge fini-dinantien et anté-stéphanien des granites bretons avaient été étendues à l'ensemble de la France de l'Ouest. Quant aux granites gneissiques des landes de Lanvaux, les recherches de A.-M. Faure-Muret et celles toutes récentes de J. Cogne (6) ne permettent guère de douter de leur âge prévarisque (orogenèse cadomienne).

Il n'en demeure pas moins que tous les auteurs - et même ceux qui mettent aujourd'hui l'accent sur le caractère progressif de la granitisation ou sur les phénomènes de régénération des granites - sont d'accord pour reconnaître à l'orogenèse hercynienne une part prépondérante, sans être exclusive, dans la mise en place des granites et des complexes cristallophylliens en Armorique et singulièrement en Armorique péninsulaire.

On est donc fondé à estimer que les formations sédimentaires cryptozoïques, rangées sous la dénomination «briovérien» et affectées du signe $X$ sur les cartes -- et au sein desquelles les venues intrusives ont monté - s'étendaient alors beaucoup plus largement qu'aujourd'hui. Ceci est d'autant plus vraisemblable que le socle n'avait pas encore subi le raccourcissement imposé par les contraintes hercyniennes.

Les schistes briovériens ou phyllades de St-Lô (Briovéra) soni des schistes argileux, de teinte verdâtre ou gris verdâtre, parfois bleutés, très fissiles, ardoisiers ou subardoisiers, au voisinage des bancs durs qui les arment (grès, conglomérats, phtanites, calcaires). Leur puissance est difficile à évaluer, car ils ont été vigoureusement plissés. Le style des plis, délicat à mettre en évidence, d'ailleurs, paraît très irrégulier (couches incompétentes). Une schistosité intense masque le plus souvent lá direction et le pendage réels des bancs.

Avant l'épreuve des mouvements hercyniens, qui a été décisive dans l'acquisition de ces caractères, il devait s'agir d'un ensemble demeuré relativement plastique, déjà induré mécani-

(6) J. Cogne : Remarques à propos d'une étude récente sur l'âge des granites de la Bretagne méridionale. C.R. Som. Soc. Géol. France, 1951, p. 276-8. 
quement par les orogenèses antérieures (cadomienne et «phase intermédiaire» mal datée ?) et localement pénétré par les roches intrusives associées à ces orogenèses pré-hercyniennes. Bref, un «socle $\triangleright$ bien différent du socle cristallin rigide évoqué plus haut.

$2^{\circ}$ La couverture paléozoĭque se caractérise par la puissanc: des assises de grès-quartzite qui alternent avec des assises de schistes souvent ardoisiers. Les grès de Ste-Suzanne, dans le Maine, le grès armoricain dans toute l'Armorique, les quartzites de Plougastel en Bretagne constituent une armature rigide dont il y a lieu de tenir compte pour comprendre le comportement mécanique de cette couverture. Les assises de schistes interstratifiées ont assurément facilité les déformations; la preuve nous en est fournie par le fait que la série paléozoïque est plissée de manière relativement souple. Mais on ne saurait oublier que les valeurs du seuil de déformations plastiques des grès sont parmi les plus élevées qui soient. J. Goguel cite l'exemple des grès rouges du Trias (qui n'ont pas, à beaucoup près, la rigidité du grès armoricain) pour lequel il avance le chiffre de $3.000 \mathrm{~kg} . / \mathrm{cm}^{2}$ : à titre de comparaison, les calcaires marneux tendres du crétacé inférieur, dans le Jura, n'exigent que $500 \mathrm{~kg}$. au $\mathrm{cm}^{2}$ et le gypse 200 seulement. Le même auteur ajoute : « Le grès massif, non interrompu par des lits marneux, est très difficilement déformable avec un seuil sans doute plus élevé que pour toute autre roche sédimentaire...»(7). N'est-ce pas le cas des puissantes assises de grès d'Armorique dont l'épaisseur peut atteindre 300 à 400 mètres ? On imagine sans difficulté la résistance offerte à la déformation par ces séries gréseuses.

$3^{\circ}$ On le conçoit d'autant mieux que la base de cette couverture est conglomératique ou gréseuse. Or un * conglomérat peut être presque aussi résistant qu'un grès si ses bancs ne sont pas séparés par des lits argileux plastiques... 》(7). Le poudingue de Rocreux, en Normandie, le poudingue de Montfort, et un poudingue à galets de quartz (faciès Gourin de la carte de Redon) que nous plaçons dans la même position stratigraphique, à la base de la série pourprée, en Bretagne centrale (8), le poudingue de Sigournais, en Vendée, le poudingue de base du grès armoricain là où ce grès est transgressif sur le socle briovérien en Normandie (région de Mortain) ou en Bretagne (Segréen), ces divers poudingues tiennent en Armorique la position occupée dans le Jura et en Afrique du Nord par les assises gypsifères et salifères du Trias.

Ils jalonnent, en outre, une vaste surface de discordance, depuis

(7) J. GogurL : Traité de Tectonique, p. 187-188-190.

(8) C. KLein : Poudingue de Gourin et poudingue de Montfort sur le pourtour de la région silurienne au S. de Rennes. Bull. Soc. Géol. Frarce, 1956. 
longtemps reconnue en Normandie, où elle se place à la base de la série cambrienne datée par les calcaires à Archoeocyathus de Carteret. Cette discordance demeure l'objet de contestations en Bretagne centrale, où les travaux de $F$. Kerforne, de $Y$. Milon, de Ed. Bolelli, ont pourtant démontré sa réalité et où elle se situerait stratigraphiquement plus haut, à la base de la série pourprée (Trémadoc ?). Cette discordance, nous l'avons, à notre tour, reconnue d'une manière très constante : elle apparait admirablement au droit des terminaisons périsynclinales de la série paléozoique et nous avons même pu la photographier (carrière de la Marette au S. de St-Malon). P. Pruvost l'étend à la Vendée où les schistes pourprés de Chantonnay, reposant sur le poudingue de Sigournais, sont considérés comme équivalents des schistes pourprés bretons (discordance vendéenne) (9).

Certes, ni F. Kerforne, ni Y. Milon n'ont considéré le contact entre les schistes $X$ et la série pourprée comme le signe d'une discordance stratigraphique, mais comme celui d'un contact anormal. Ed. Bolelli, par contre, y a reconnu une discordance stratigraphique véritable mais ultèrieurement modifiée par les mouvements tectoniques; et il a employé pour la définir une expression que nous lui emprunterons bien que nous ne puissions accepter son interprétation mécanique : celle de discordance tectonisée (10).

Ainsi les mouvements hercyniens, très puissants, se sont exercés sur un socle schisteux modérément plissé et très imparfaitement induré, injecté localement par des venues intrusives (en Normandie et en Bretagne méridionale, notamment). Sur ce socle arasé par l'érosion reposait en discordance une série sédimentaire puissamment armée de bancs de grès-quartzites, d'une grande rigidité mécanique, et débutant par un conglomérat de base d'une rigidité sensiblement comparable. Telles sont les données originales du problème; elles sont, on le voit, fort différentes des données classiques.

\section{III. - L'ÉPREUVE DEs CONTRAINTES MÉcanigues DE L'OROGENÈSE HERCYNIENNE}

Les effets de l'orogenèse hercynienne se sont inscrits dans cette structure originale d'une manière assez explicite pour que Yinterprétation qui va en être suggérée puisse à tout instant se référer à des données d'observation précises.

$1^{\circ}$ Raccourcissement et induration du socle briovérien. Le raccourcissement du socle est incontestable puisque les schistes

(9) P. Pruvost : Le Cambrien du Massif Armoricain. Congrès Géol. Int., Mexico, 1956.

(10) Ed. Bolell : Contribution à l'estude tectonique de la région synclinale au S. de Rennes. Mém. de la Soc. Géol. et Min. de Bret., t. IX, 1951. 
briovériens ont été fortement plissés et comprimés. Il s'agit de savoir ce qui, dans ce plissement et cette schistosité, revient aux orogenèses pré-hercyniennes et à l'orogenèse hercynienne ellemême. Celle-ci est, pour l'essentiel, responsable du plissement de la couverture discordante dont nous établirons, plus loin, la solidarité mécanique avec le socle. On peut donc considérer que la contraction de la couverture paléozoique donne une valeur minima du raccourcissement du socle au cours de l'orogenèse hercynienne.

G. Mathieu estime qu' * il y a environ $300 \mathrm{~km}$. entre Caen (discordance de Rocreux) et Niort (série continue du Saunort) $\gg$ et que * cette distance était au moins trois fois plus grande avant les plissements hercyniens... (11). C'est donc d'un raccourcissement des $2 / 3$ qu'il s'agirait. Les plis du socle, tronqués par la surface de discordance, ont rejoué sous la couverture à la manière d'un soufflet qu'on referme sous une feuille de carton. Ils se sont accentués et les anciennes directions tectoniques ont été réaffirmées. En même temps, une schistosité puissante apparaissait, qui, théoriquement, devrait nous permettre de définir le sens des compressions mécaniques responsables (12). Il ne serait pas surprenant qu'on puisse faire apparaître un désaccord, au sein des schistes $X$, entre la direction des plis hérités des déformations pré-hercyniennes et celle des plans de schistosité issus des contraintes hercyniennes. Mais on ne dispose pas, pour en discuter, des éléments de base indispensables.

On peut penser, par contre, que la plasticité des phyllades a permis le rejeu de la trame-accordéon des plissements pré-hercyniens. Par conséquent, le raccourcissement réel du socle sous l'effet des compressions hercyniennes serait très supérieur aux valeurs de la seule contraction de la couverture.

Dans le même temps, les venues éruptives syntectoniques montaient à travers la série phylladienne et pénétraient souvent (mais non toujours, ni partout) au sein de la couverture ellemême. Si la Bretagne péninsulaire nous offre d'admirables exemples de la structure dite * Pacifique $\gg$, la Normandie (réserve faite du granite de Flamanville) et le Maine paraissent avoir été granitisés avant la mise en place de la couverture cambro-silurienne.

$2^{\circ}$ Schistosité et plissement de la couverture. On conviendra qu'un tel raccourcissement du socle n'a pu rester sans effet sur

(11) G. Mathieu : Le probleme du Précambrien dans l'Ouest de la France. La Revue Scientifique, n॰ 3.241 , fév. 1945.

(12) J. Goover : * Les auteurs anglais ont reconnu que la schistosité apparait suivant l'un des plans principaux de l'ellipsoide de déformation : celui qui est perpendiculaire a la direction de compression maximum... La schistosité est perpendiculaire, en chaque point, a la direction d'écrasement maximum... Traité de Tectonique, p. 41 . 
la couverture discordante. Nous examinerons plus loin comment ces contraintes horizontales - car elles paraissent avoir été prépondérantes en l'occurrence - se sont exprimées au niveau de la surface de discordance et comment on peut concevoir la solidarité mécanique du socle et de sa couverture.

La vigueur du serrage a fait apparaitre dans les schistes de la couverture, et parfois même dans les grès, une extraordinaire schistosité. Tous les auteurs ont souligné le désaccord fondamental et constant qui existe entre l'inclinaison des plans de schistosité et celle des plans de stratification. On l'observe, d'une manière très apparente, dans les schistes pourprés qui affleurent au milieu des landes, au S. de Rennes, ou, plus démonstratif encore, au droit des terminaisons périsynclinales de la couverture. (La Chicaudière au S.-E. du Rocher d'Uzel). Cette schistosité subverticale existe même au sein des assises demeurées subhorizontales (Forêt de Paimpont et camp de Coëtquidam). Elle nous parait être un argument non négligeable à l'appui de notre hypothèse de contraintes tangentielles transmises à la couverture par le socle en voie de raccourcissement (fig. 1).
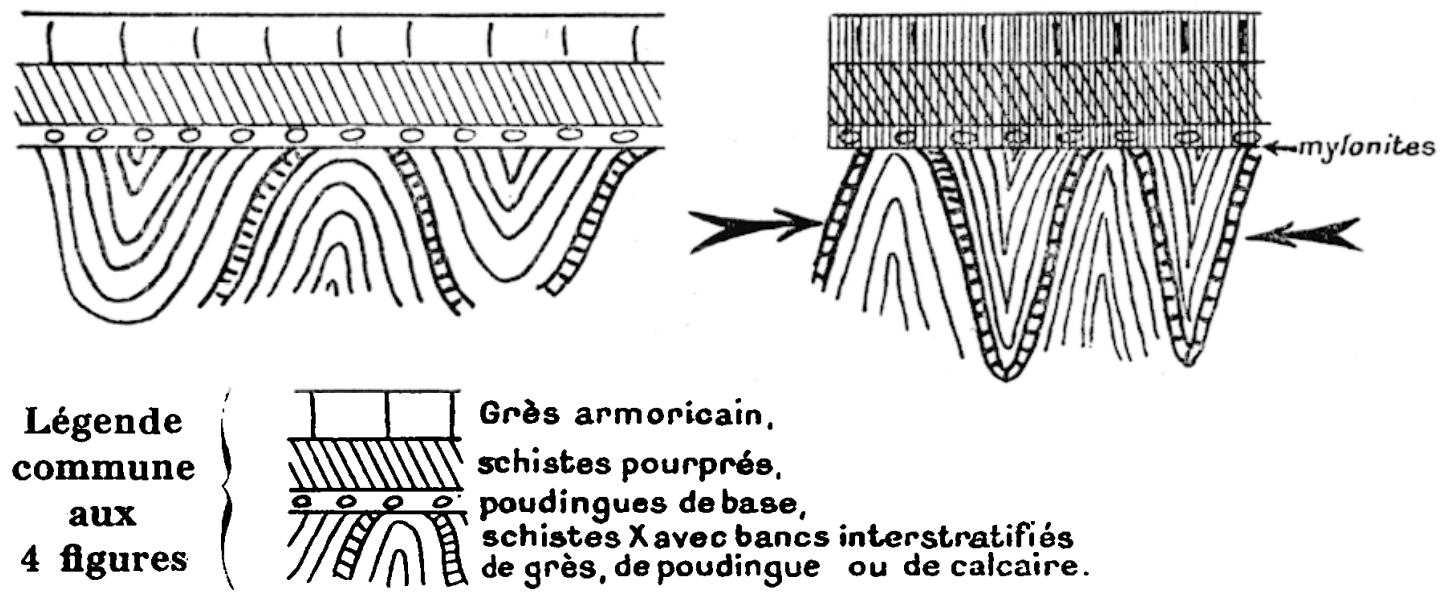

Fra. 1

Cependant, l'apparition de la schistosité et les modifications corrélatives de la structure intime des roches, ne pouvaient suffire $\grave{a}$ absorber tous les effets de cette compression du socle. La couverture, grâce à la présence des assises schisteuses et malgré la rigidité des grès-quartzites, pouvait se plisser pourvu que les sollicitations mécaniques fussent suffisamment énergiques (13). Le rythme et le style de ses plis ne sont d'ailleurs pas uniformes :

(13) On peut douter que les forces résultant des seuls effets de la gravité soient suffisantes pour avoir pu déformer une couverture aussi puissamment armée et au sein de laquelle, les assises schisteuses elles-mêmes sont souvent schisto-gréseuses. 
ils expriment en tous lieux les conditions locales du comportement du socle.

- Dans la Forêt de Paimpont, les schistes pourprés et les grès armoricains sont restés subhorizontaux (fig. 1).

- De part et d'autre du cours de la Vilaine, les ondulations de la couverture sont amples et régulières (fig. 2 ).

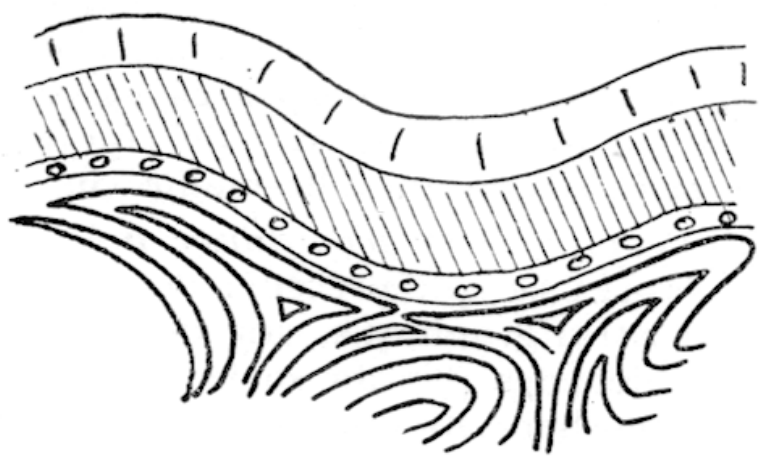

Fig. 2

- Dans le Segréen, un régime de plis étroits et profonds apparaît.

En Normandie, les synclinaux sont souvent dissymétriques et leur flanc $\mathbf{N}$ est laminé.

- En Vendée, les plis hercyniens dessinent des $V$ très accusés, passant même à des plis en $Y$ par étirement du fond (fig. 3 et 4).

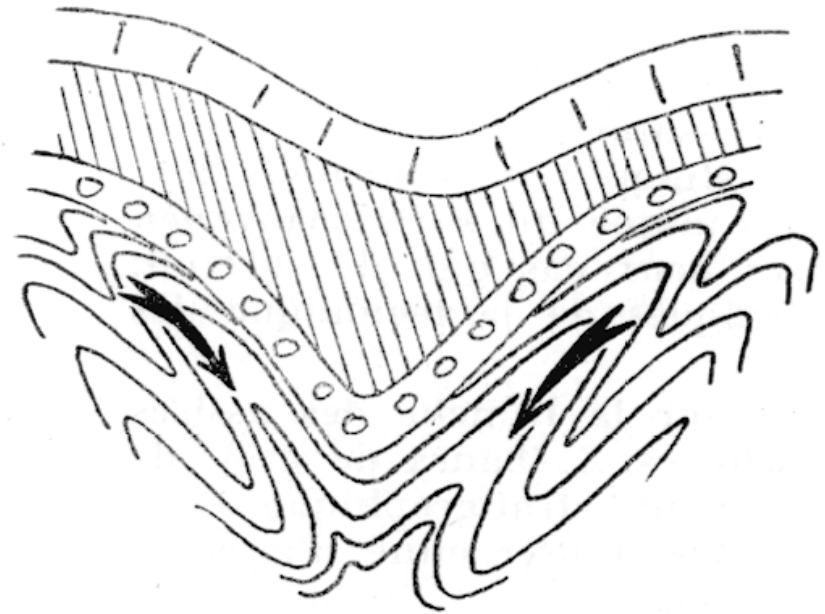

Fic. 3

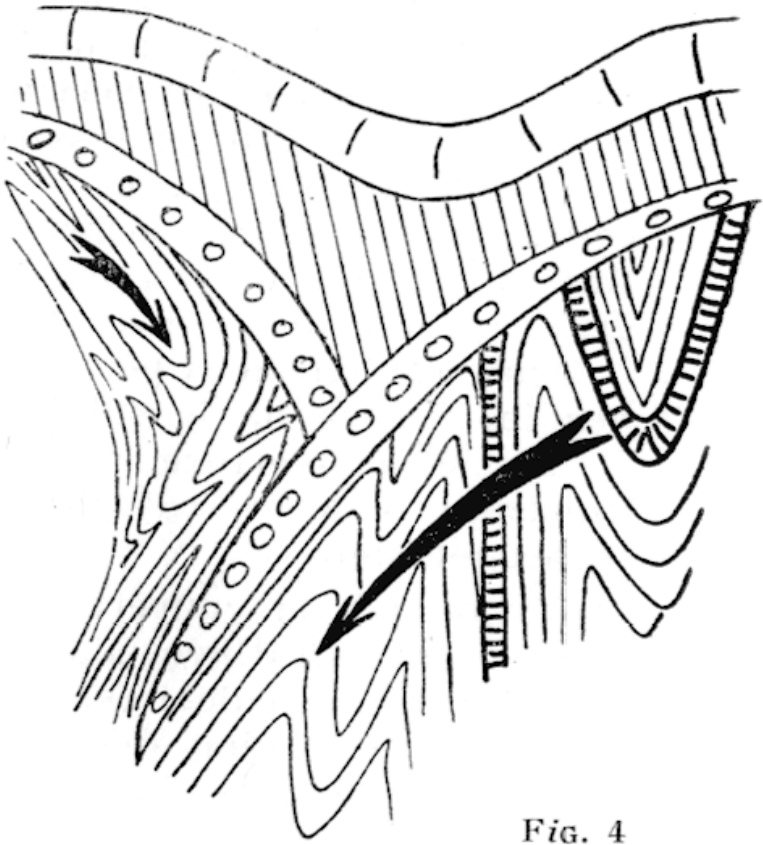

Fig. 4 
Les singularités tectoniques notées par A. Bigot en Normandie ou par G. Mathieu en Vendée s'expliquent de façon séduisante par la dynamique même du mode de plissement. La tectonique de couverture armoricaine est une expression très originale de la tectonique du socle schisteux sous-jacent. Nous pensons que les contraintes tangentielles ont joué un rôle décisif dans l'acquisition de la schistosité et dans la mise en place des plis de la couverture paléozoïque. C'est le sous-entraînement de cette couverture sous l'effet du rejeu des plis-accordéon tronqués du substratum qui nous paraît le mieux rendre compte des caractères de ces tectoniques superposées et cependant étroitement solidaires. C'est du reste la vue de coupes naturelles qui nous a suggéré cette dynamique elle-même (fig. $1,2,3,4$ ).

$3^{\circ}$ Discordance tectonisée et accordance mécanique. On serait peut-être tenté, d'après ce qui précède, de concevoir au niveau de la discordance stratigraphique, un décollement général de la couverture. Le rejeu des plis du socle, leur accentuation sous l'effet des contraintes hercyniennes seraient responsables de ce décollement et impliqueraient l'indépendance mécanique de la couverture par rapport au socle. En somme, on se trouverait placé dans une situation comparable à celle rencontrée dans le Jura, les Préalpes du Nord ou le Maghreb. Il n'en est rien.

D'une part, le décollement, lorsqu'il existe, se place au niveau même de la discordance stratigraphique. C'est cette surface de discontinuité stratigraphique qui est devenue une surface de discontinuité tectonique et non tel ou tel niveau plastique plus ou moins voisin de la surface de discordance.

D'autre part, ce n'est pas l'écoulement des masses sous l'effet de la gravité qui provoque la disharmonie. Il ne faut pas oublier la différence fondamentale que nous avons soulignće entre la nature du socle briovérien, schisteux, demeuré relativement souple - beaucoup plus souple assurément que sa couverture à base conglomératique et à armature gréseuse puissante - et celle, cristalline, des socles hercyniens surmontés d'une couverture plastique que la tectonique d'écoulement suffit à plisser grâce à l'interposition du Trias gypso-salin.

Deux cas extrêmes peuvent être distingués qui vont nous donner la mesure de la solidarité mécanique du socle briovérien et 'de sa couverture paléozoïque ; deux cas qui, de surcroît, illustrent bien les caractères spécifiques de la tectonique des socles schisteux.

a) Lorsque la couverture repose sur la tranche des assises rigides interstratifiées dans les schistes $X$ (bancs de grès, de poudingues, de calcaires), la discordance stratigraphique a été tectonisée. Le rejeu des plis du socle a déterminé d'intenses frictions au long de la surface de discordance et des mylonites - témoins assurés de la vigueur des frottements -, jalonnent 
la base rigide de la couverture. Le laminage partiel ou total de l'assise des poudingues de base est la conséquence extrême de ce jeu de la discordance tectonisée. La petite carrière de la Marette illustre parfaitement le cas qui vient d'être décrit. A St-Thurial, il est possible que nous soyons en présence du même phénomène.

b) Par contre, lorsque la couverture reposait sur la tranche des bancs de schistes argileux, ceux-ci se sont ployés souplement sous l'assise rigide des conglomérats ou des grès discordants, en pseudo-concordance. F. Kerforne, Y. Milon, Ed. Bolelli ont cité et décrit d'innombrables exemples de pseudo-concordance qui sont à l'origine - Ie fait n'est guère douteux — des prises de position de nombreux géologues sur le caractère concordant de la série briovérienne et de la série pourprée bretonne.

Nous pensons que ce phénomène d'accordance, d'une grande généralité, justifie amplement une étroite solidarité mécanique entre le socle et sa couverture. Celle-ci a pu être entraînée et impliquée dans un rythme de déformation très particulier dont les plis en $V$ ou en $Y$ ne représentent que des cas extrêmes et non des rnoins suggestifs.

\section{Conclusion}

Il n'était pas question, dans le cadre d'un article aussi bref, d'envisager dans le détail une question aussi vaste. Nous nous proposons d'y revenir ailleurs (14). Mais nous avons voulu dégager un mode de comportement original du socle vis-à-vis de sa couverture. La nature du matériel (surtout schisteux), un certain héritage mécanique (l'épreuve des orogenèses pré-hercyniennes) justifient les réactions du socle briovérien. La rigidité de certaines assises de la couverture, et singulièrement des assises de la base, le fait que cette couverture, mécaniquement vierge, reposait en discordance sur le socle lors de l'orogenèse hercynienne, permettent de comprendre ses réactions au cours de la contraction du socle. La schistosité subverticale qui est apparue au sein de la série discordante, le rythme et le style des plis qui l'ont affectée, nous incitent à voir dans les contraintes tangentielles, transmises par le socle, le moteur principal des déformations.

C'est de ce comportement original que serait issue la structure appalachienne si admirablement exprimée dans le relief au $S$. de Rennes. C'est de cette même histoire qu'est héritée la riche trame tectonique (d'innombrables failles de décrochement au long des flancs synclinaux) qui joue un rôle si grand dans le tracé du réseau hydrographique et singulièrement dans le détail de son dessin. On le voit, l'étude morphologique de la région ne saurait être tentée sans une connaissance préalable de sa tectonique originale.

(14) CI. KLEIN : Quelques caractères originaux du socle armoricain. Nonors (à paraitre). 